\title{
Mineral deposition and vascular invasion of hydroxyapatite reinforced collagen scaffolds seeded with human adipose- derived stem cells
}

Holly E. Weiss-Bilka ${ }^{1}$, Matthew J. Meagher ${ }^{1}$, Joshua A. Gargac ${ }^{2}$, Glen L. Niebur ${ }^{1,3}$, Ryan K. Roeder ${ }^{1,3}$ and Diane R. Wagner ${ }^{4^{*}}$ (D)

\begin{abstract}
Background: Collagen-based scaffolds reinforced with hydroxyapatite (HA) are an attractive choice for bone tissue engineering because their composition mimics that of bone. We previously reported the development of compression-molded collagen-HA scaffolds that exhibited high porosity, interconnected pores, and mechanical properties that were well-suited for surgical handling and fixation. The objective of this study was to investigate these novel collagen-HA scaffolds in combination with human adipose-derived stem cells (hASCs) as a template for bone formation in a subcutaneous athymic mouse model.

Methods: Collagen-HA scaffolds and collagen-only scaffolds were fabricated as previously described, and a clinically approved bone void filler was used as a control for the material. Constructs were seeded with hASCs and were pretreated with either control or osteogenic media. A cell-free group was also included. Scaffolds were implanted subcutaneously in the backs of athymic nude mice for 8 weeks. Mineral deposition was quantified via microcomputed tomography. Histological and immunofluorescence images of the explants were used to analyze their vascular invasion, remodeling and cellularity.

Results: Cell-free collagen-HA scaffolds and those that were pre-seeded with osteogenically differentiated hASCs supported mineral deposition and vascular invasion at comparable rates, while cell-seeded constructs treated with the control medium showed lower mineralization after implantation. HA-reinforcement allowed collagen constructs to maintain their shape, provided improved cell-tissue-scaffold integration, and resulted in a more organized tissue when pre-treated in an osteogenic medium. Scaffold type and pre-treatment also determined osteoclast activity and therefore potential remodeling of the constructs.

Conclusions: The results of this study cumulatively indicate that treatment medium and scaffold composition direct mineralization and angiogenic tissue formation in an ectopic model. The data suggest that it may be necessary to match the scaffold with a particular cell type and cell-specific pre-treatment to achieve optimal bone formation.
\end{abstract}

Keywords: Bone, Osteogenesis, Angiogenesis, Adipose-derived stem cell, Collagen, Hydroxyapatite, Scaffold, Ectopic

\footnotetext{
* Correspondence: wagnerdi@iupui.edu

${ }^{4}$ Department of Mechanical and Energy Engineering, Indiana

University-Purdue University Indianapolis, 723 W. Michigan Ave SL260,

Indianapolis, IN 46202, USA

Full list of author information is available at the end of the article
}

(c) The Author(s). 2019 Open Access This article is distributed under the terms of the Creative Commons Attribution 4.0 International License (http://creativecommons.org/licenses/by/4.0/), which permits unrestricted use, distribution, and reproduction in any medium, provided you give appropriate credit to the original author(s) and the source, provide a link to the Creative Commons license, and indicate if changes were made. The Creative Commons Public Domain Dedication waiver (http://creativecommons.org/publicdomain/zero/1.0/) applies to the data made available in this article, unless otherwise stated. 


\section{Background}

Non-union fractures and critical size bone defects have a considerable impact upon the global population [1]. In fact, bone is the second most transplanted tissue worldwide with an estimated 2.2 million graft procedures performed each year [2]. Yet despite their prevalence, autogenic grafts are limited by the availability of donor tissue and are often associated with donor site pain, while allogenic grafting techniques carry the risk of morbidity and infection [3]. These limitations have inspired many research efforts involving laboratory-produced tissue replacements; however current approaches to bone tissue engineering generally lack a sufficient functionality compared to native bone matrix.

Collagen-based scaffolds reinforced with hydroxyapatite (HA) are an attractive choice for bone tissue engineering because they mimic the key components of bone, collagen and mineral, and possess improved mechanical properties compared to either component alone [4, 5]. We previously reported novel compression-molded collagen-HA scaffolds that exhibited high porosity (85$90 \%), ~ 300-400 \mu \mathrm{m}$ interconnected pores, struts composed of high-density collagen fibrils reinforced with HA whiskers, and mechanical properties that were wellsuited for surgical handling and fixation [6]. These scaffolds were also conducive to the infiltration and in vitro differentiation of adipose-derived stem cells [6]. After ectopic implantation, the vascular density, cell density, matrix deposition, and micro-computed tomography (micro-CT) bone volume increased with increasing HA content in the scaffolds [7]. However, in these previous studies, the collagen-HA scaffolds were implanted without pre-seeding with osteogenic cells, which may further increase bone tissue generation.

Human adult adipose-derived stem cells (hASCs) are an appealing complement to such scaffolds because they are abundant and have been shown to contribute to both bone formation and vasculogenesis [8] in vivo. Subcutaneous implantation in immunodeficient mice is an established model for evaluating combinations of hASCs with various scaffolds and culture conditions, with multiple reports of successful bone formation in the literature [9]. One of the earliest successful studies coupled $\beta$-tricalcium phosphate discs with hASCs and then pre-treatment in an osteoinductive medium for 2 weeks. During 8 weeks of subcutaneous implantation in nude mice, the cellseeded discs developed an osteocalcin-rich tissue containing osteoclasts and infiltrated by blood vessels [10]. Another study reported bone formation in 4 of 5 HA-tricalcium phosphate (TCP) and in 1 of 5 Collagraft $^{\ominus}$ (collagen-HA-TCP composite matrix) scaffolds that had been seeded with untreated hASCs and subjected to 6 weeks subcutaneous implantation in nude mice [11]. In a different investigation, hASCs seeded onto porous HA ceramic scaffolds and cultured in a 3-D perfusion system for 5 days prior to subcutaneous implantation in nude mice for 8 weeks resulted in well vascularized constructs containing osteoprogenitor cells and positive immunostaining for human bone sialoprotein [12]. Interestingly, immunostaining for human CD31 and CD34 indicated that newly formed vessels were of human origin. More recently, the importance of surface topography was demonstrated in the context of $\mathrm{TiO}_{2}$ nanotube surfaces, which enhanced the osteogenic differentiation of hASCs both in vitro and in vivo [13]. Finally, hASCs were first cultured on either extracellular matrix derived from bone marrow-derived mesenchymal stem cells or tissue culture plastic before being loaded on HA powder and implanted subcutaneously in immunodeficient mice; hASCs that had been expanded on the cell-derived matrix produced more bone tissue compared to those cultured on tissue culture plastic [14].

The objective of this study was therefore to investigate the use of novel collagen-HA (CHA) scaffolds in combination with hASCs as a template for bone formation in a subcutaneous athymic mouse model. Cell-seeded constructs were pre-treated with either control medium $(\mathrm{CM})$, or an osteogenic medium (OM). A cell-free, or acelluar (Acel), control group of collagen-HA scaffolds was included to assess the osteoinductive capacity of the scaffold itself. This group was cultured in OM for the same duration as cell-seeded groups. The effect of HA in the scaffold was examined by including a collagenonly $(\mathrm{Col})$ control group. Finally, $\mathrm{NuOss}^{\mathrm{Tm}}(\mathrm{Nu})-\mathrm{a}$ clinically approved bone void filler previously reported to support bone formation in ectopic models when coupled with human periosteum-derived cells, human mesoangioblasts and a murine pre-chondrogenic cell line [15, 16] - was included as a control for the scaffold material.

\section{Methods \\ Cell culture}

Human adipose-derived stem cells (ZenBio, Durham, $\mathrm{NC}$ ) derived and pooled from the subcutaneous adipose tissue of 5 non-diabetic female donors were expanded as previously described $[17,18]$. During expansion, cells were plated at a density of 3000 cells $/ \mathrm{cm}^{2}$ and were maintained in DMEM/F12 medium (MediaTech, Herndon, VA) containing 10\% FBS (Atlas Biologicals, Fort Collins, CO), 1\% Penicillin-Streptomycin (Pen-Strep, MediaTech), $5 \mathrm{ng} / \mathrm{mL}$ human epidermal growth factor (hEGF), $1 \mathrm{ng} / \mathrm{mL}$ human fibroblast growth factor-2 (hFGF2) and $0.25 \mathrm{ng} / \mathrm{mL}$ transforming growth factor- $\beta 1$ (TGF- $\beta 1)$ [19]; all growth factors from PeproTech, 
Rocky Hill, NJ). Cells were treated with fresh medium every 2 days.

\section{Scaffold preparation}

Collagen $(\mathrm{Col})$ and collagen with 40 vol\% hydroxyapatite whiskers (CHA) scaffolds with $85 \%$ porosity and a mean pore size of $\sim 375 \mu \mathrm{m}$ were fabricated as previously described $[6,7]$. NuOss ${ }^{\text {Tw }}$ scaffolds were purchased from Ace Surgical Supply (Brockton, MA). All scaffolds were sized to $21 \mathrm{~mm}^{3}$ ( $3 \mathrm{~mm}$ diameter $\times 3 \mathrm{~mm}$ height) using a sterile biopsy punch. After crosslinking and porogen leaching of Col and CHA scaffolds, implants were sterilized by submersion in 70\% ethanol and rehydrated in sterile PBS followed by sterile culture medium supplemented with $1 \%$ Pen-Strep.

\section{Cell seeding and in vitro culture}

For cell seeding, pre-sterilized scaffolds were transferred to a sterile gauze pad, where excess medium was removed, and then to sterile, agarose-coated tissue culture plates. Passage 7 hASCs were drop-seeded onto each scaffold in a $20 \mu \mathrm{L}$ suspension at a seeding density of $21 \times 10^{6}$ cells $/ \mathrm{mL}$ and placed into a humidified incubator at $37^{\circ} \mathrm{C}$ and $5 \% \mathrm{CO}_{2}$ for 1 hour to attach. After 1 hour, $1 \mathrm{~mL}$ of either control medium (CM: DMEM-HG, $10 \%$ FBS, 1\% Pen-Strep) or osteogenic differentiation medium $[10,20]$ (OM: CM supplemented with $50 \mu \mathrm{g} /$ $\mathrm{mL}$ ascorbic acid, $10 \mathrm{mM} \beta$-glycerophosphate, $0.1 \mu \mathrm{M}$ dexamethasone; all additives from Sigma-Aldrich, St. Louis, MO) was added to each construct. $\mathrm{NuOss}^{\text {Tm }}$ scaffolds, previously successful in inducing bone formation with human periosteal-derived cells in an ectopic model [15], were included as a control. These scaffolds are of bovine origin and contain natural bone mineral in an open collagen network. Preliminary studies demonstrated that no bone was formed when this scaffold was implanted without cells. Collagen-only (Col) scaffolds were included to assess the contribution of HA whiskers to bone formation, and the importance of preimplantation osteogenic induction was investigated by treating seeded hASCs with OM compared to CM.

Following overnight incubation, the culture medium was collected from each well containing a cell-seeded scaffold and unattached cells were pelleted at $300 \times g$, resuspended, and counted with a hemocytometer to determine seeding efficiency. Fresh culture medium was added to all scaffolds three times per week for 14 days, which is the time at which hASC monolayer cultures have previously shown markers of osteogenic differentiation in vitro [11, 20]. ALP activity and calcium nodules were confirmed in cells grown in monolayer and treated with $\mathrm{OM}$ and $\mathrm{CM}$ for 14 days. On the day of implantation, 1 scaffold per group (see Table 1) was rinsed with PBS, fixed overnight in $4 \%$ paraformaldehyde at $4{ }^{\circ} \mathrm{C}$, embedded in optimal cutting temperature (OCT) compound and processed for histology.

\section{Subcutaneous ectopic implantation in mice}

Remaining scaffolds were implanted subcutaneously in the cervical region of athymic nude mice (Harlan Laboratories, Indianapolis, IN). All procedures were carried out in compliance with protocols approved by the Institutional Animal Care and Use Committee (IACUC) of the University of Notre Dame. Mice were anesthetized with a 'rodent cocktail' consisting of $100 \mathrm{mg} / \mathrm{mL}$ Ketamine, $20 \mathrm{mg} / \mathrm{mL}$ Xylazine and $10 \mathrm{mg} / \mathrm{mL}$ Acepromazine (all from Henry Schein, Dublin, $\mathrm{OH}$ ) in sterile saline, according to the following dosage $[6,7]$ : Volume anesthetic $[\mu \mathrm{L}]=($ Body weight $[\mathrm{g}] \times 10)-50$. Three constructs from three different groups were implanted in each mouse (five mice total) through a small incision in the center of the dorsal region. Following 8 weeks of implantation, mice were sacrificed and scaffolds were recovered.

\section{Micro-computed tomography of scaffolds and explants}

Prior to cell seeding, all scaffolds to be implanted were scanned by micro-CT (Bioscan NanoSPECT/CT, Mediso Medical Imaging Systems, Budapest, Hungary) at $10 \mu \mathrm{m}$ resolution, $70 \mathrm{kVp}$ voltage, $100 \mathrm{~mA}$ current with 720 projections at $600 \mathrm{~ms}$ integration time. Micro-CT images were median filtered to reduce noise. The bone volume (BV) was measured by segmenting images at a threshold of 1900 , which corresponded to $294 \mathrm{mg} \mathrm{HA} / \mathrm{cm}^{3}$ using a custom calibration phantom [21]. An ex-vivo micro-CT scan was performed on all explants after overnight fixation in $4 \%$ paraformaldehyde, using the same parameters as the pre-implantation scans. De novo mineralization was measured by the difference in thresholded BV between the implant and explant.

Table 1 Overview of experimental groups

\begin{tabular}{llllll}
\hline Abbreviation & Nu OM & Col OM & CHA Acel & CHA CM & CHA OM \\
\hline Scaffold & NuOss & Collagen/HA \\
Calcium source & CaP granules & Collagen & Collagen/HA & Collagen/HA & HA whiskers \\
hASCs & + & + & HA whiskers & + & HA whiskers \\
Medium & OM & OM & OM & CM & OM \\
\hline
\end{tabular}




\section{Histology and immunofluorescence}

Fixed samples were rinsed with PBS, decalcified in a 0.5 $M$ EDTA solution and subjected to a series of increasing concentrations of sucrose in PBS. Explants were equilibrated in OCT compound (Sakura, Torrance, CA) for 3 $\mathrm{h}$, frozen in dry ice-cooled isopentane, and stored at $80^{\circ} \mathrm{C}$ prior to sectioning. Each embedded sample was cryosectioned at a thickness of 7-9 $\mu \mathrm{m}$ and transferred to gelatin subbed slides, which were dried at $37^{\circ} \mathrm{C}$ for 2 $\mathrm{h}$ and then stored at $-80^{\circ} \mathrm{C}$. Slides were warmed to room temperature and dried prior to all staining procedures. Sections were stained with H\&E following standard histological techniques. Tartrate resistant acid phosphatase (TRAP) staining was performed by $2 \mathrm{~h}$ incubation in TRAP buffer $(50 \mathrm{mM}$ sodium acetate, 30 $\mathrm{mM}$ sodium tartrate, $0.1 \%$ Triton $\mathrm{X}-100, \mathrm{pH} 5$ ). The buffer was then replaced with TRAP stain for $1 \mathrm{~h}$ before 2 rinses in PBS, counterstaining with hematoxylin and mounting with aqueous mounting medium [16].

All immunofluorescence (IF) procedures were optimized for the specific antibody. Unless otherwise noted, IF procedures were performed for CD31 (R\&D Systems; $10 \mu \mathrm{g} / \mathrm{mL}$ ), osteopontin (OPN goat polyclonal, R\&D Systems; $15 \mu \mathrm{g} / \mathrm{mL}$ ), vascular endothelial growth factor (VEGF rabbit polyclonal, Abcam; $1 \mu \mathrm{g} / \mathrm{mL}$ ), osteocalcin (OCN rabbit polyclonal, Abcam; 1:1000) as follows. Slides were brought to room temperature, hydrated in PBS and, when necessary, subjected to antigen retrieval consisting of $5 \mathrm{~min}$ incubation at $90^{\circ} \mathrm{C}$ in $1 \mathrm{mM}$ EDTA, $0.05 \%$ Polysorbate-20, $\mathrm{pH}$ 8.0. Sections were cooled to room temperature, rinsed with $\mathrm{PBS}$, and blocked with $1 \%$ normal serum from the animal in which the secondary antibody was raised and $0.3 \mathrm{M}$ glycine in PBS for 30 min at room temperature. Primary antibody was applied in blocking solution overnight at $4{ }^{\circ} \mathrm{C}$. Slides were rinsed with PBS before the secondary antibody (Alexa Fluor ${ }^{\circ}$ anti-goat IgG and anti-rabbit IgG, Invitrogen; 1:200) was diluted in $1 \%$ BSA and applied for $1 \mathrm{~h}$ at room temperature. Finally, sections were counterstained with 4',6-Diamidino-2-Phenylindole (DAPI), rinsed with PBS, and mounted in an aqueous medium.

For human nuclei antibody (HNA), sections were brought to room temperature, permeabilized with ice cold acetone for $10 \mathrm{~min}$ at room temperature, dried and re-hydrated in PBS. Blocking was performed in two steps: first with $3 \%$ normal donkey serum and $0.3 \mathrm{M}$ glycine in PBS for $30 \mathrm{~min}$, and then with Mouse IgG Blocking Reagent (Vector Labs, Burlingame, CA). Primary antibody was applied (1:200, Mouse Monoclonal 235-1 IgG1, Rockland, Gilbertsville, PA) at room temperature for $1 \mathrm{~h}$, sections were rinsed with PBS, and the secondary antibody was applied for $20 \mathrm{~min}$ at room temperature (Alexa Fluor ${ }^{\circ} 488$ Donkey anti-mouse, Invitrogen; 1: 800). All nuclei were counterstained with DAPI (Vector
Labs), and endogenous fluorescence was quenched with a $1 \mathrm{~min}$ Trypan blue incubation $(250 \mu \mathrm{g} / \mathrm{mL}, \mathrm{pH} 4.4$, Invitrogen, Grand Island, NY) [22]. Sections were rinsed quickly with PBS, and mounted with aqueous medium.

\section{Quantification and analysis}

To quantify the extent of vascular invasion into each group, 6 randomized images were taken from H\&Estained sections of each scaffold. The number of vessels, identified as lumina containing erythrocytes, was counted for each image, and 18 images (6 per scaffold; 3 randomized images per section at 2 depths) were averaged for each group and the number of vessels per $\mathrm{mm}^{2}$ were reported [23].

Cellularity was evaluated by quantifying the number of cells in DAPI-stained sections. Images were taken at central and peripheral radial locations of a given crosssection, at shallow and mid-height depths for each construct. The calculated number of cells from the center and periphery of a given cross-section were summed and normalized by the total imaged area to obtain a total number of cells per $\mathrm{mm}^{2}$. Images were taken at 400X magnification and processed in MATLAB (MathWorks, Natick, MA) with CellC [24, 25], applying a segmentation factor of 0.9 and cell shape as the segmentation modality.

Statistical analyses were performed in Prism (GraphPad, La Jolla, CA). Significance was assessed by 1-way or 2 -way ANOVA and Tukey's post-hoc test $(p<0.05)$ as appropriate. Data are presented as the mean \pm SEM.

\section{Results}

Cell seeding and scaffold preparation

Following the initial attachment period and overnight incubation, cell-containing constructs had a minimum seeding efficiency of $99.33 \pm 0.11 \%$ (Fig. 1a). Histological analysis of scaffolds cultured for 14 days in vitro verified cellular infiltration throughout all scaffold types and a developing extracellular matrix in the pore spaces (Fig. 1b-e). Cells appeared to be more evenly distributed in $\mathrm{Col}$ and CHA scaffolds compared to NuOss ${ }^{\mathrm{Tm}}$ controls, where cells were more densely populated around the periphery of the scaffolds. NuOss ${ }^{\text {Tux }}$ (Fig. 1b) and Col (Fig. 1c) controls exhibited a non-circular cross-section and volumetric shrinkage after 2 weeks of culture, whereas CHA scaffolds cultured in either type of treatment medium (Fig. 1d, e) maintained their original size and shape.

Cells grown in monolayer and treated with $\mathrm{OM}$ and CM stained positive for alkaline phosphatase activity and contained small nodules of mineralization after 14 days of induction (not shown). The intensity and incidence of staining was higher for cells cultured with OM, 

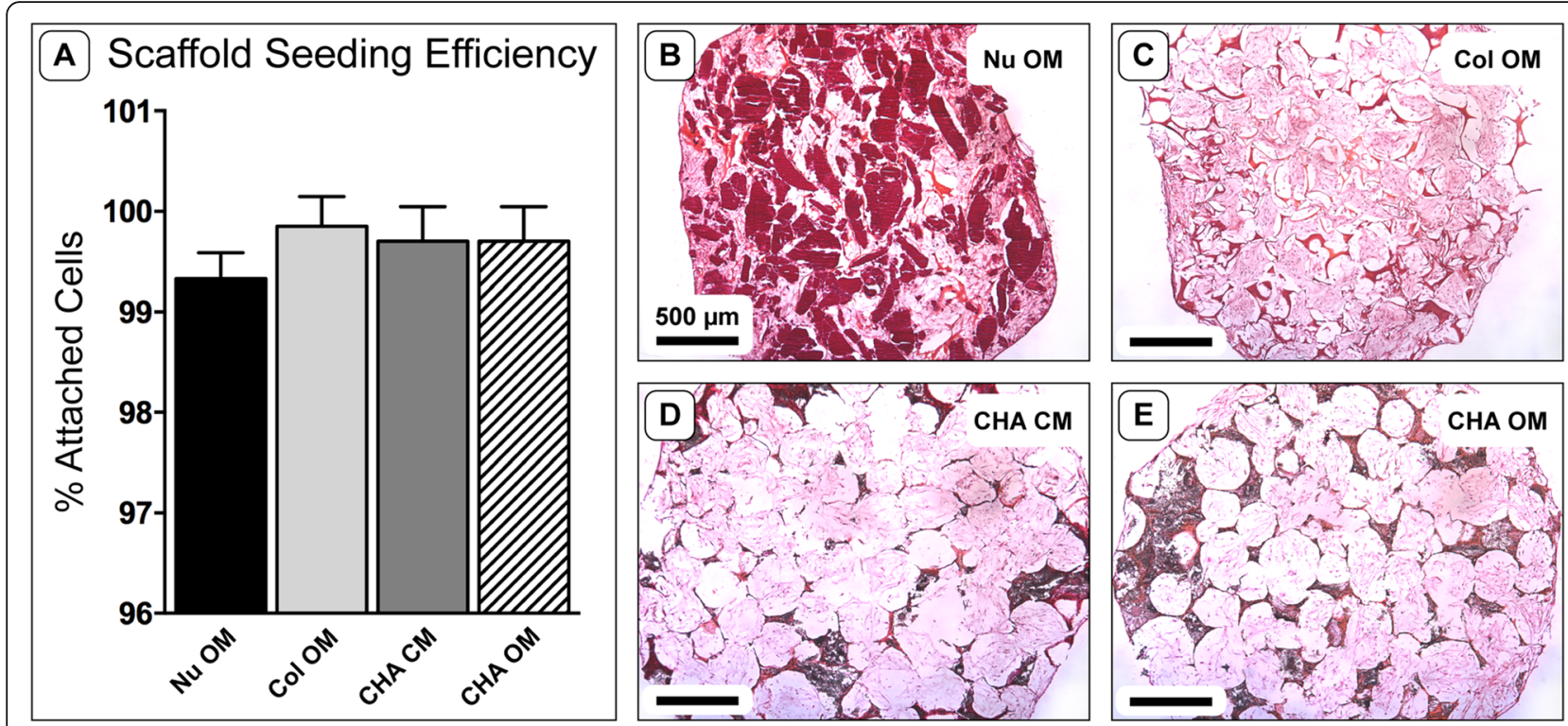

Fig. 1 Scaffold seeding efficiency and morphology after in vitro culture. a Scaffold seeding efficiency, one-way ANOVA $(n=4 ; p=0.17)$. b-e Representative H\&E stained cryosections of hASC-seeded scaffolds after 14 days in culture. Note the composition of each scaffold by coloration: dark red indicates calcium phosphate granules in NuOss ${ }^{\mathrm{TM}}$ scaffolds; red-orange indicates collagen; black indicates the presence of HA whiskers; small, dark spots indicate cell nuclei; light pink indicates ECM deposited by hASCs; white space represents empty pore space

indicating that this treatment was directing hASCs toward an osteogenic lineage.

Tissue morphology and micro-CT analysis

Gross morphological evaluation revealed that $\mathrm{Col}$ and CHA CM explants were noticeably smaller than implanted constructs, and $\mathrm{NuOss}^{\mathrm{Tx}}$ constructs were white, while all other groups had a light-pink tint (Fig. 2).
Blood vessels were most prominent on $\mathrm{CHA} \mathrm{OM}$ and CHA Acel explants; however, vessels were also visible in $\mathrm{CHA} \mathrm{CM}$ and $\mathrm{Col}$ explants. Interestingly, no blood vessels were visible in the $\mathrm{NuOss}^{\mathrm{Tt}}$ control group.

After 8 weeks implantation, explants exhibited varying degrees of bone formation as determined by micro-CT. Each group exhibited a significant change between preimplant ( 0 week) and post-explant ( 8 week) measurements

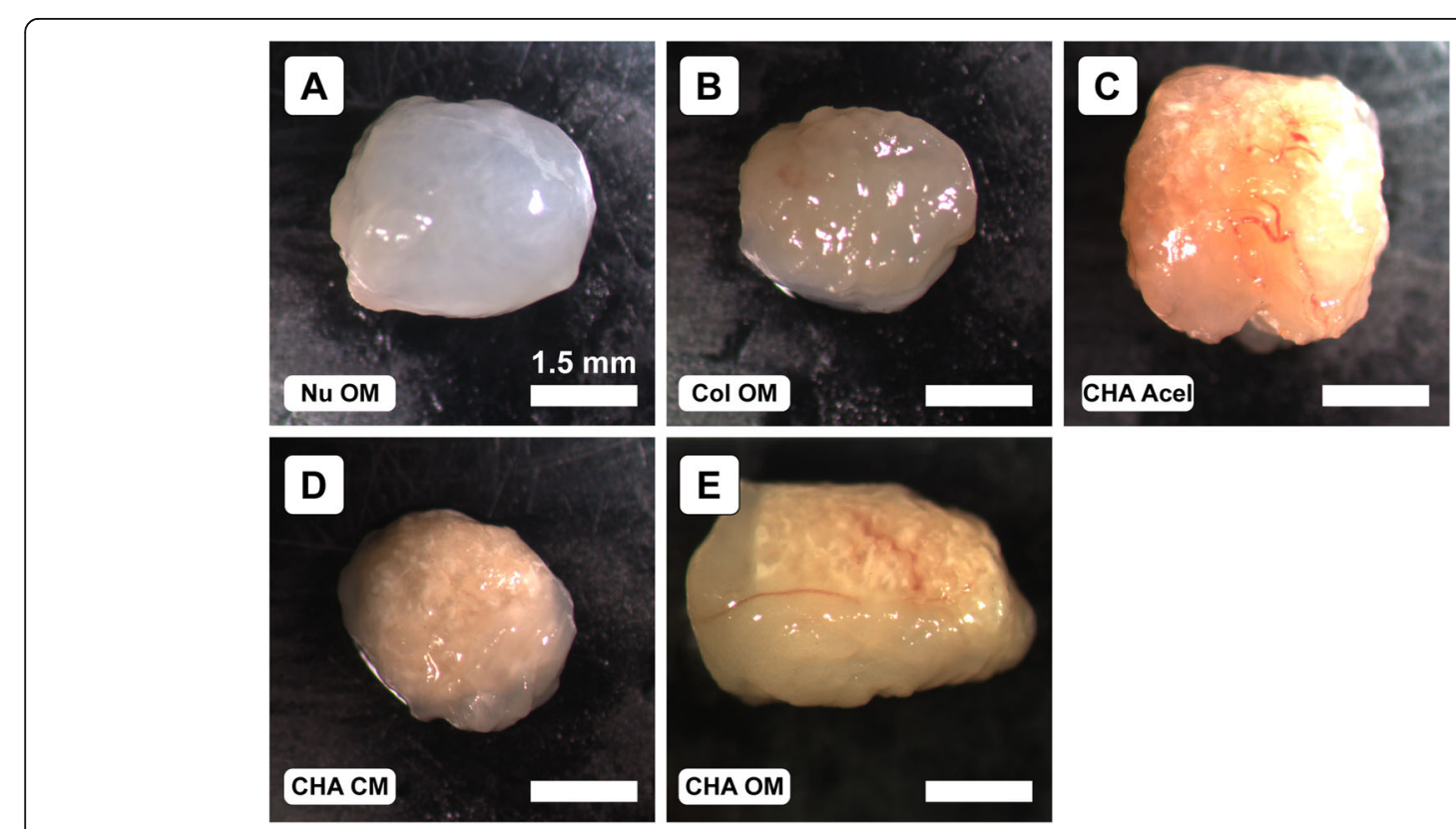

Fig. 2 Representative gross morphology of explants (a-e) after 8 weeks implantation 
of bone volume (Fig. 3a). NuOss ${ }^{\text {Th }}$ controls decreased in measured bone volume by $3.05 \pm 0.55 \mathrm{~mm}^{3}$. In contrast, the bone volume of $\mathrm{Col} \mathrm{OM}$ constructs increased from 0 to $1.06 \mathrm{~mm}^{3}$, and $\mathrm{CHA} \mathrm{OM}$ constructs increased in volume by $2.08 \pm 0.21 \mathrm{~mm}^{3}$. Interestingly, acellular CHA scaffolds increased in bone volume by $2.14 \mathrm{~mm}^{3}$, while the CHA CM constructs decreased by $2.40 \pm 0.11 \mathrm{~mm}^{3}$.

$H \& E$ stained sections revealed substantial differences in tissue morphology among groups. Like pre-implantation samples, the central region of $\mathrm{NuOss}^{\mathrm{Tw}}$ scaffolds appeared to be less cellular than the periphery, based upon the eosin stain intensity (Fig. 3b). Tissue infiltration in Col scaffolds (Fig. 3c) was not as dense as in the CHA scaffolds (Fig. 3d-f). CHA OM and CHA Acel scaffolds maintained a well-defined porous structure, while the pore structure of $\mathrm{CHA} \mathrm{CM}$ and $\mathrm{Col} \mathrm{OM}$ constructs appeared deformed and partially collapsed. Any changes in the porosity of $\mathrm{NuOss}^{\mathrm{TM}}$ scaffolds were less clear, but a certain amount of scaffold remodeling was apparent via tissue infiltration into the calcium phosphate granules.

Higher magnification revealed the organizational structure of the tissues within each group. Interestingly, treatment medium appeared to elicit a distinct tissue morphology and cellular response in hASC-seeded CHA scaffolds. CHA CM constructs formed a dense, disorganized tissue (Fig. 3e); whereas CHA OM constructs produced a spatially organized tissue (Fig. 3f). In general, the dense tissue observed in CHA OM constructs was preferentially located around the periphery of pore spaces and formed a ring-like structure around less dense tissue in the center of the pores. The tissue within CHA Acel constructs was similarly organized (Fig. 3d). Dense, eosinophilic tissue was also observed at the edges of tissue within pore space in Col OM constructs; however, there were typically gaps between this tissue and the walls of the collagen struts (Fig. 3c).

\section{Osteogenic and vasculogenic markers}

Immunofluorescence staining revealed osteocalcinpositive tissue located in the matrix of $\mathrm{CHA}$ Acel and OM groups (Fig. 4c, e). Positive staining was also observed to a lesser extent in $\mathrm{NuOss}^{\mathrm{Tm}}$, $\mathrm{Col}$ and CHA CM groups (Fig. 4a, b, d). Osteopontin immunofluorescence was more intense for HA-containing constructs, and appeared to localize to the scaffold structure (Fig. 4h-j). Interestingly, the spatial patterning of osteopontin was similar in $\mathrm{NuOss}^{\mathrm{Tm}}$ and $\mathrm{Col}$ groups, though the staining intensity was not as strong as in constructs that contained HA (Fig. 4f, g).

Histological analysis revealed the degree of vascularization for each group (Fig. 5). CHA OM constructs had a higher blood vessel count $(23.0 \pm 3.3$
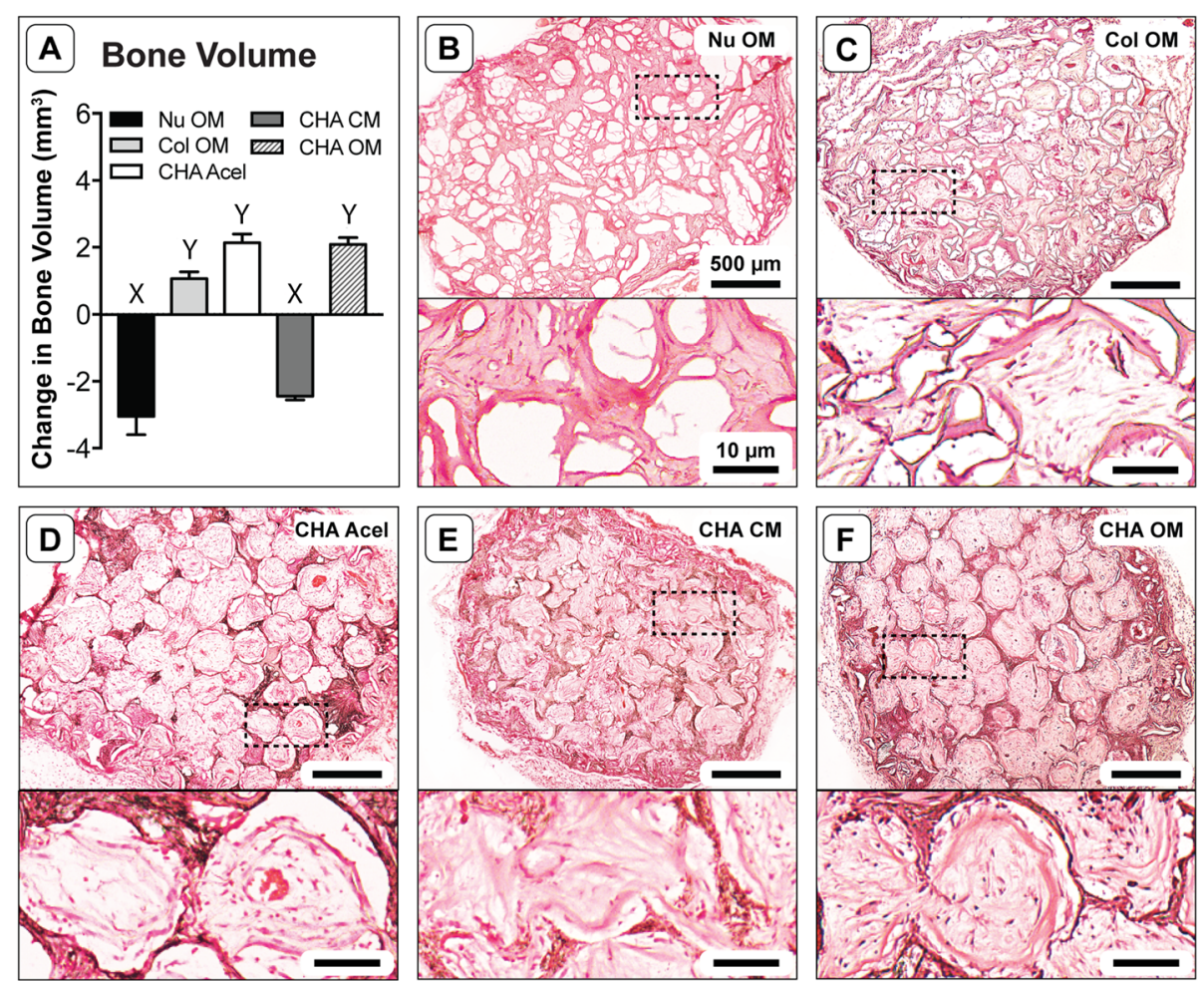

Fig. 3 Change in bone volume and tissue morphology after 8 weeks implantation. a Absolute change in bone volume as measured by micro- $C T$; oneway ANOVA and Tukey's post-hoc test $(n=3 ; p<0.0001)$. Different letters indicate statistically significant differences. b-f Representative H\&E stained cryosections showing the entire scaffold cross-section (upper image) and new tissue within scaffold pore spaces at higher magnification (lower image) 

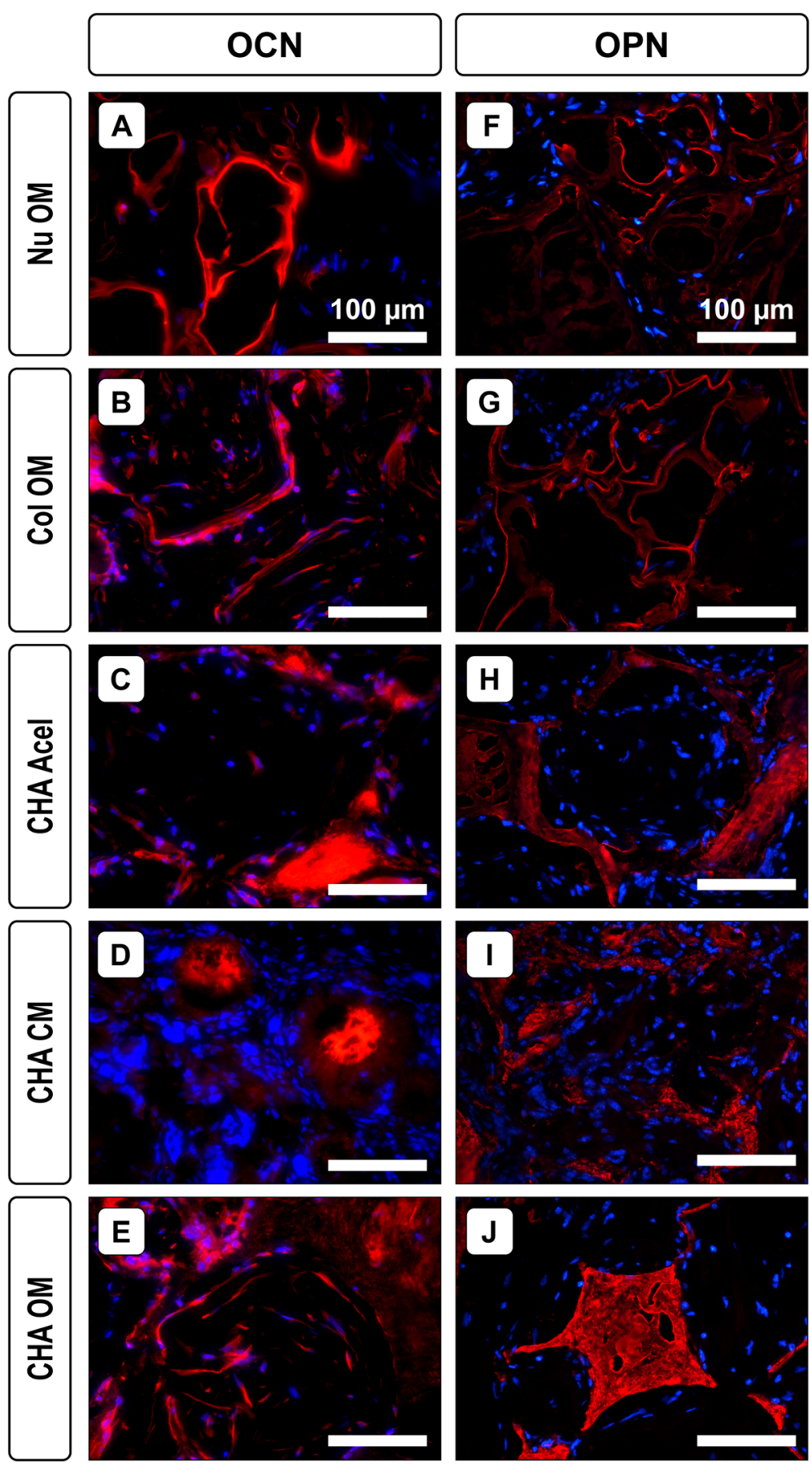

Fig. 4 Osteogenic markers in explanted constructs. Representative immunostained sections showing (a-e) osteocalcin and (f-j) osteopontin after 8 weeks implantation. Red: osteocalcin or osteopontin; blue: DAPI (cell nuclei)

vessels $\left./ \mathrm{mm}^{2}\right)$ than CHA CM $\left(13.8 \pm 2.1\right.$ vessels $\left./ \mathrm{mm}^{2}\right)$ and $\mathrm{NuOss}^{\mathrm{Tu}}\left(5.0 \pm 1.0\right.$ vessels $\left./ \mathrm{mm}^{2}\right)$ constructs. Col and CHA Acel also had significantly more vessels than $\mathrm{NuOss}^{\mathrm{Tu}}$ scaffolds at $18.4 \pm 1.8$ and $16.8 \pm 2.2$ vessels per $\mathrm{mm}^{2}$, respectively.

To better understand differences in vascularization, VEGF levels in the cell-seeded scaffolds were also visualized via immunofluorescence. Within in vitro constructs, hASCs were actively secreting VEGF after 14 days of culture (Fig. 6a-e, Pre-implant). Immunostaining for VEGF was more apparent in OM groups at this time point. Images of post-implantation constructs displayed a different trend (Fig. 6f-j, Post-implant). CHA OM and CHA Acel groups had a high level of intense staining, 

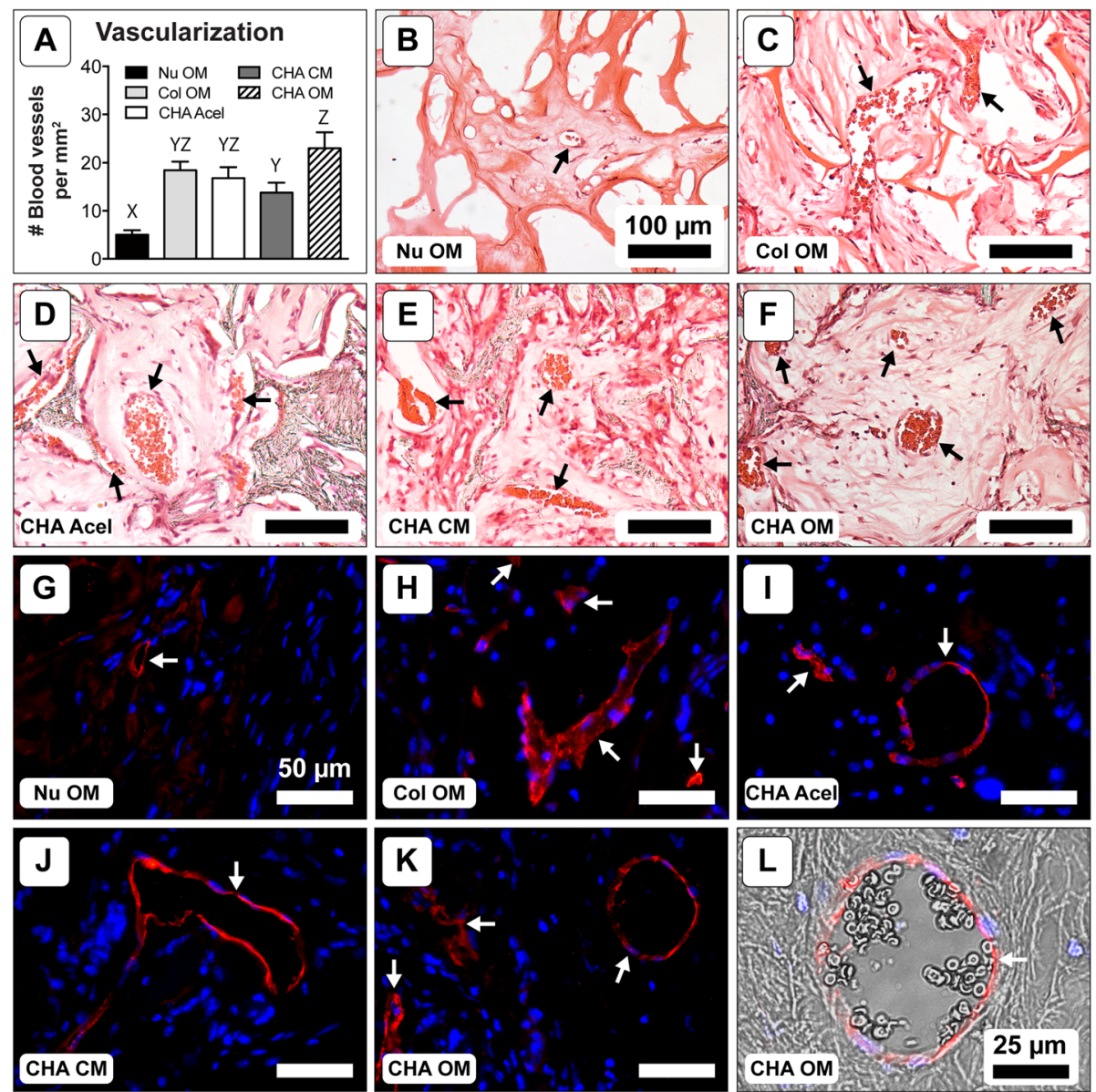

Fig. 5 Vascularization after 8 weeks implantation. a Blood vessel density; one-way ANOVA and Tukey's post-hoc test $(n=18 ; p<0.05)$. Different letters indicate statistically significant differences. b-f Representative H\&E stained cryosections showing blood vessels (black arrows). (G-K) Representative immunofluorescent stained sections showing CD31 (red) with DAPI counterstain (blue). White arrows denote blood vessels. (L) CD31 (red) and DAPI (blue) immunofluorescent staining overlaid on a matched bright field image showing the presence of erythrocytes within the vessel lumen

while CHA CM constructs maintained a low level of VEGF. $\mathrm{Nu}$ OM constructs, which exhibited levels of VEGF staining comparable to CHA OM scaffolds after in vitro culture, exhibited a marked decrease in VEGF expression after 8 weeks of implantation. Staining was similar at both time points for the Col OM group; however, the intensity of the stain was lower than that of CHA Acel and CHA OM groups after 8 weeks of implantation.

\section{Osteoclast activity and cellularity}

Tartrate-resistant acid phosphatase (TRAP) staining demonstrated that there was considerable osteoclast activity in CHA CM constructs (Fig. 7). Positive activity was also identified on the periphery of $\mathrm{NuOss}^{\mathrm{TM}}$ and, to a lesser extent, CHA OM explants. No staining was detected in Col or CHA Acel groups.

The cellularity of each explant was also evaluated (Fig. 8a). Comparing cell counts from the cross-sections sampled, the number of cells in constructs cultured in vitro for 14 days was not statistically different (Fig. $8 \mathrm{~b})$. However, following 8 weeks in vivo, $\mathrm{NuOss}^{\mathrm{Tx}}$ explants contained fewer cells and CHA CM explants contained more cells than all other groups (Fig. 8b), with 8 week $\mathrm{NuOss}^{\mathrm{Tw}}$ explants containing approximately the same number of cells as the 14 days in vitro time point. The cell distribution of evaluated cross-sections was not significantly different for Col OM and CHA CM constructs; however, the number of cells quantified at the center of CHA OM cross-sections was lower than at the periphery for in vitro scaffolds and for 8 week constructs (Fig. 8b). Unlike $\mathrm{NuOss}^{\mathrm{Tw}}$ explants, however, the cellularity of the CHA OM group increased by a factor of 4.5 following implantation.

Human nuclei immunofluorescence was used in conjunction with DAPI to evaluate the donor cell contribution to new tissue formation in explants. Human cells were identified within the implanted construct in all 


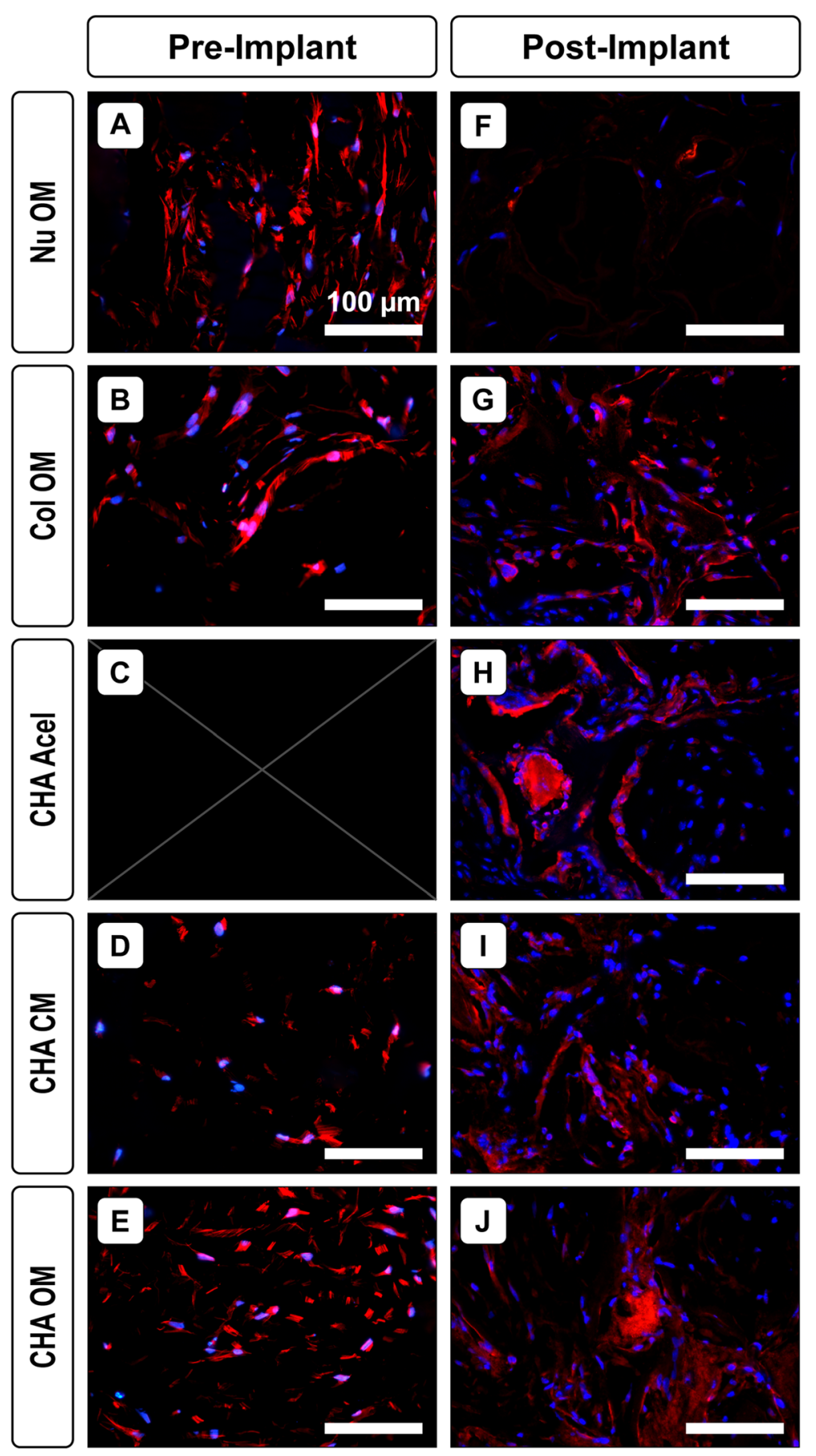

Fig. 6 VEGF levels before and after implantation. Representative sections showing VEGF (red) and cell nuclei (blue) (a-e) after 14 days of in vitro culture but before implantation and $(\mathbf{f}-\mathbf{j})$ after 8 weeks implantation

cell-seeded scaffold types (Fig. 9); however, few remained after 8 weeks of implantation. The hASCs detected were primarily located on the periphery of the scaffolds or in the surrounding tissue. Additionally, despite physical separation within the mice, a single hASC was detected in the surrounding tissue of a CHA Acel explant (data not shown).

\section{Discussion}

The results of this study cumulatively indicate that treatment medium and scaffold composition direct mineralization and angiogenic tissue formation in an ectopic model. One important effect of HA reinforcement in collagen-based carriers was evident after only 14 days of culture in vitro. All constructs with $\mathrm{CHA}$ scaffolds 

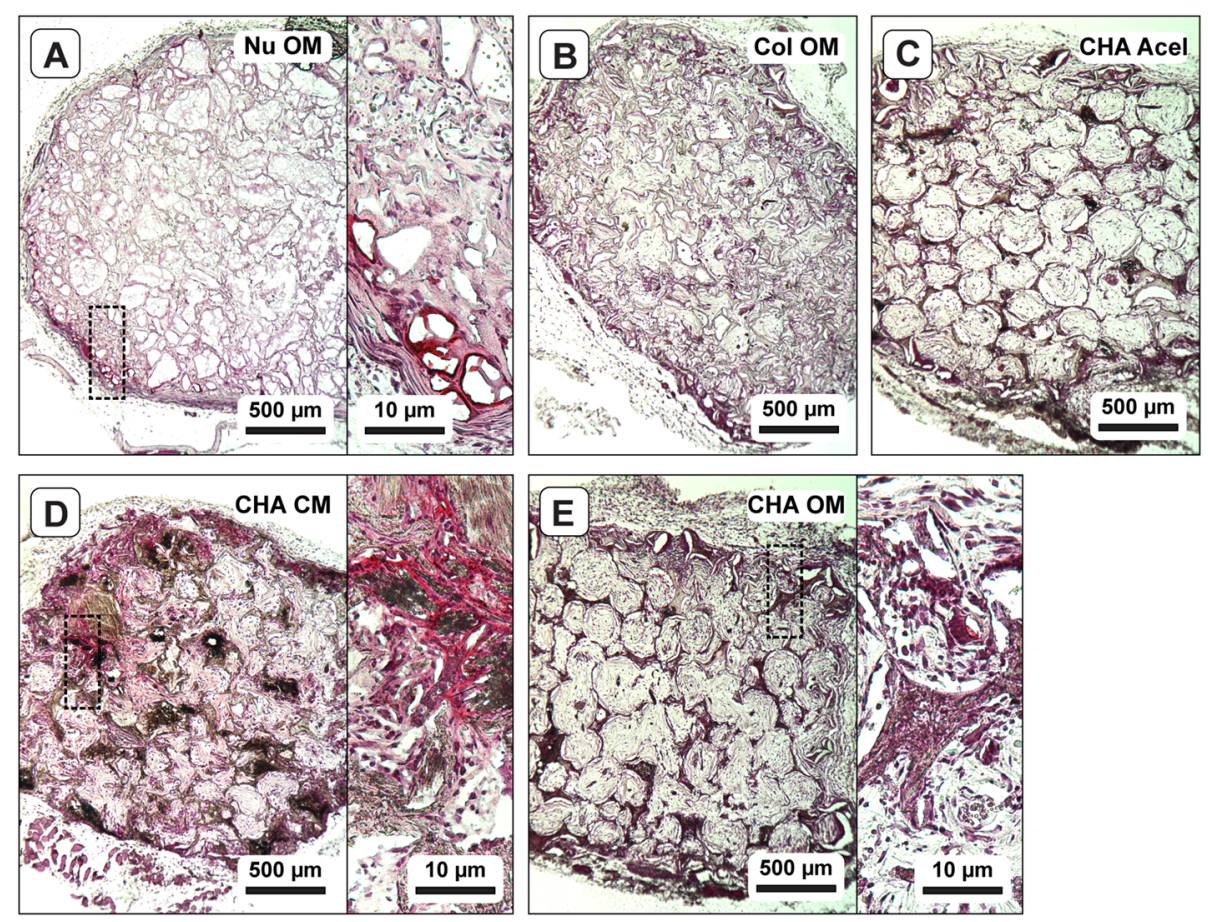

Fig. 7 Osteoclast activity in explanted constructs (a-e). Representative TRAP-stained sections showing osteoclast activity (red)

maintained the original size and circular cross-section throughout the pre-treatment period, whereas $\mathrm{NuOss}^{\mathrm{Tu}}$ and collagen scaffolds exhibited volumetric shrinkage into an irregular, oblong-like shape. This finding suggests that HA whisker reinforcement increased the structural stability of collagen-based scaffolds throughout in vitro culture.

In terms of mineralization, the mineral content measured by micro-CT was increased in cell-seeded CHA scaffolds with pre-treatment in $\mathrm{OM}$, but the increase was not significantly different from that in acellular CHA scaffolds. Similar results have been observed previously for other osteoinductive scaffolds [26, 27]. Lyons et al. reported that matrix deposited by MSCs during in vitro culture may adversely affect healing by acting as a barrier to macrophage-mediated remodelling when implanted in vivo [28]. The current study confirms previous reports that the CHA scaffolds alone promote the recruitment and osteogenic activity of endogenous cell populations [6, 7]. Additionally, ASC-seeded $\mathrm{NuOss}^{\mathrm{Tn}}$


Fig. 8 Cell distribution in scaffolds pre- and post-implantation. a Scaffold cross-section stained with DAPI. b Radial distributions of cells in in vitro samples $(14 \mathrm{~d}, n=3)$ and 8 week explants $(8 \mathrm{w}, n=6)$. * indicates a significant difference between the periphery (black) and center (white) of individual scaffold groups $(p<0.05)$. \#\# indicates a significant increase in total cell number compared to 14d groups and $8 \mathrm{~W}$ Nu OM ( $p<0.001)$. Letters indicate that cell number for periphery $(p)$ or center $(c)$ significantly increased from $14 \mathrm{~d}$ to $8 \mathrm{w}(p<0.05)$ 

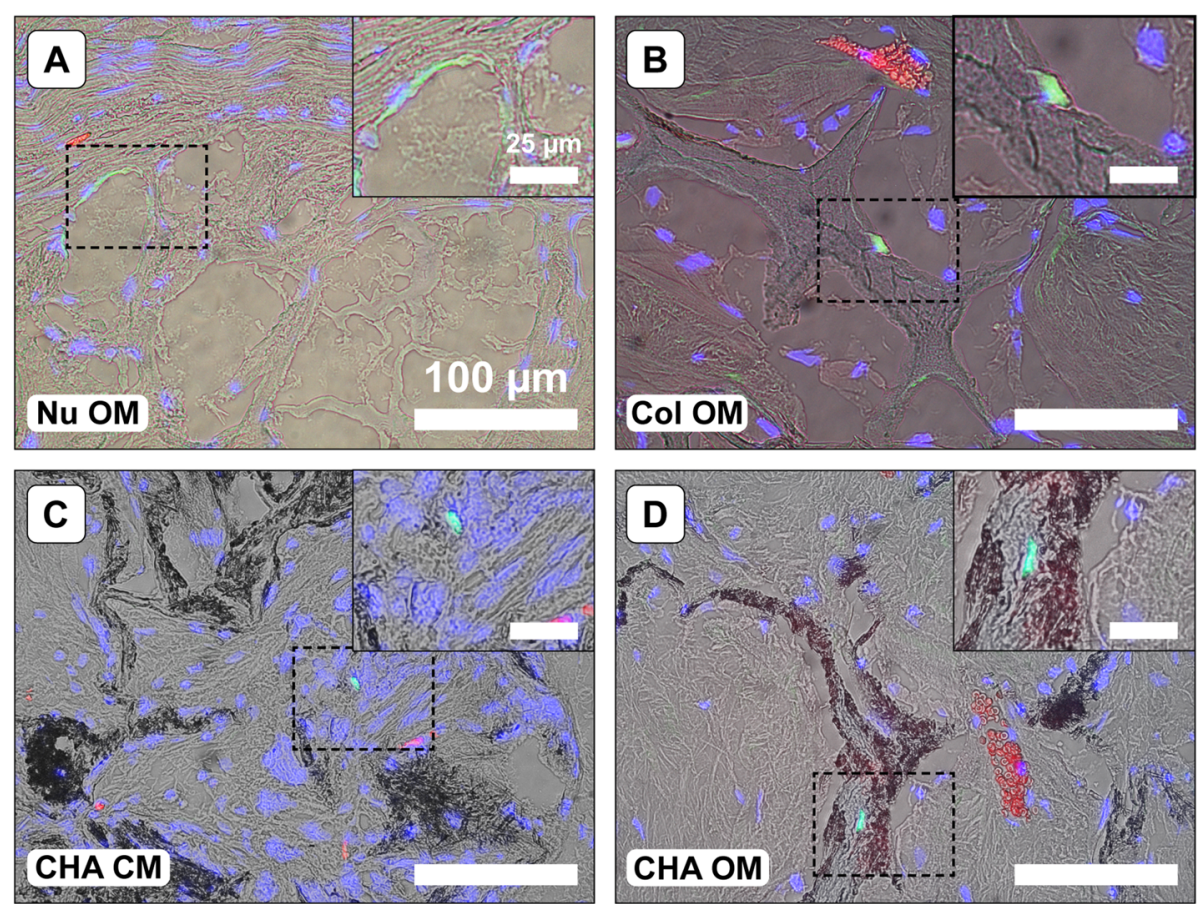

Fig. 9 Detection of human cells within explanted constructs (a-e). Representative immunofluorescent stained sections showing human nuclei antigen (green) and cell nuclei counterstained with DAPI (blue) overlaid on a matched bright field image demonstrating the location of cells within the scaffolds. Blood vessels appear in red. The locations of magnified inset regions are indicated by black dotted lines

scaffolds exhibited a decrease in bone volume, suggesting that any bone formation was insufficient to counterbalance the resorption of the scaffold. Collectively, these data suggest that a combination of cell type and pretreatment may need to be determined for a particular scaffold to achieve optimal bone regeneration.

While pre-treatment of hASC-seeded CHA scaffolds in $\mathrm{OM}$ resulted in significant increases in mineralization, pre-treatment in CM resulted in decreased mineral content. This may be a result of the high osteoclastic activity observed via TRAP staining in the CHA CM scaffolds (Fig. 7d). As opposed to mature osteoblasts, preosteoblasts have been reported to express higher levels of RANKL, which allows for the maturation, differentiation and activation of osteoclasts [29]; this may explain the high osteoclast activity in the scaffolds with undifferentiated cells in the current study. Osteoclast activity can be a positive indicator of scaffold resorption; however, the rate of resorption must be balanced with the deposition of replacement tissue and the maintenance of mechanical integrity.

To better characterize the tissue infiltrating the scaffolds, two bone markers were investigated: osteocalcin, a marker of osteoblasts that is associated with mineralized bone matrix; and osteopontin, a non-collagenous protein that is secreted by osteoblasts, osteocytes and osteoclasts and is therefore believed to play a role in both mineralization and bone remodeling [30]. Qualitatively, there was more osteopontin in CHA constructs than in collagen and $\mathrm{NuOss}^{\text {Thx }}$ scaffolds after 8 weeks of subcutaneous implantation. Localization of this protein to the scaffold structure is likely due to its ability to bind HA [30], and may account for the spatial organization of the tissue within CHA Acel and CHA OM groups. The majority of dense, bone-like matrix indicated by highly eosinophilic tissue (Fig. 3) and concentrated osteocalcin staining (Fig. 4) was identified at the periphery of the pore spaces in these constructs. HA may be at least partially responsible for this effect, as gaps were observed between the collagen-only scaffold and the extracellular matrix in the pores, and osteopontin levels were lower in these scaffolds.

Following implantation, the level of vascular invasion was higher in Col OM and CHA OM groups compared with $\mathrm{CHA} \mathrm{CM}$ constructs, indicating that osteogenic pre-treatment may have contributed to this effect. Interestingly, CHA Acel controls achieved levels of vascularization comparable to $\mathrm{CHA} \mathrm{OM}$ and $\mathrm{Col} \mathrm{OM}$ constructs, while $\mathrm{NuOss}^{\text {Tw }}$ controls contained significantly fewer vessels. A similar trend was observed in VEGF levels: staining was stronger for $\mathrm{CHA}$ OM than for CHA CM, and there was considerably less VEGF detected in $\mathrm{Nu} \mathrm{OM}$ constructs. VEGF, which is generally thought of as a key mediator of angiogenesis [31], also 
has the ability to regulate the recruitment and activity of osteoblasts, osteoclasts, and endothelial cells [32]. Therefore, higher levels of VEGF detected in CHA OM compared to CHA CM constructs both pre- and postimplantation may partially explain the differential mineralization and vascular invasion between these groups.

The tissue organization observed in CHA Acel and $\mathrm{OM}$ constructs was not maintained in $\mathrm{CHA} \mathrm{CM}$ or $\mathrm{Col}$ OM tissues in the current study (Figs. 1 and 3). For CHA CM scaffolds, this may be a result of the high osteoclastic activity observed via TRAP staining (Fig. 7). The collapsed architecture apparent in the CHA CM group, along with a high level of osteoclast activity, were indicative of rapid resorption in these scaffolds. Decreased tissue organization in Col OM scaffolds could also be the result of collapsed pore structure, likely an effect of the absence of HA.

The decreased mineral content observed in $\mathrm{NuOss}^{\mathrm{Tm}}$ scaffolds may be a result of its inhibited cellular infiltration compared to other groups. This is likely related to their 9\% lower porosity compared to Col and CHA scaffolds, as reported by the manufacturer. The eosinophilic-rich, VEGF-, osteocalcin- and osteopontinpositive tissue in this group was observed only at the scaffold periphery, where the cell density was highest. This region coincided with the location of observed blood vessels and osteoclast activity. Conversely, the central region had a very low cell density both pre- and post-implantation, and bone markers were not detected in this area. Previous studies with hASCs have reported that cell density has a significant impact upon resulting tissue formation [33], indicating that a lower concentration of cells at the scaffold center may have influenced its bone forming capacity.

Despite significant differences in vascularity, mineralization and cellularity in cell-seeded groups, few human cells were identified in any of the explants. Remaining hASCs were primarily located at the periphery of the scaffold, or in the surrounding tissue, perhaps indicating their migration out of the constructs. This hypothesis is supported by the detection of a human cell in the tissue surrounding an acellular construct. Both retention [33] and loss [34] of ASCs has been reported in the literature, introducing further confusion regarding their role in ectopic bone formation.

\section{Conclusions}

The results of this study indicate that both scaffold type and pre-treatment are crucial to successful mineral deposition and vascular invasion, and that to achieve optimal mineralization it may be necessary to match the scaffold with a particular cell type and cell-specific pretreatment. HA-reinforcement allowed collagen constructs to maintain their implanted shape, provided for improved cell-tissue-scaffold integration, and resulted in a more organized tissue when pre-treated in an osteogenic induction medium.

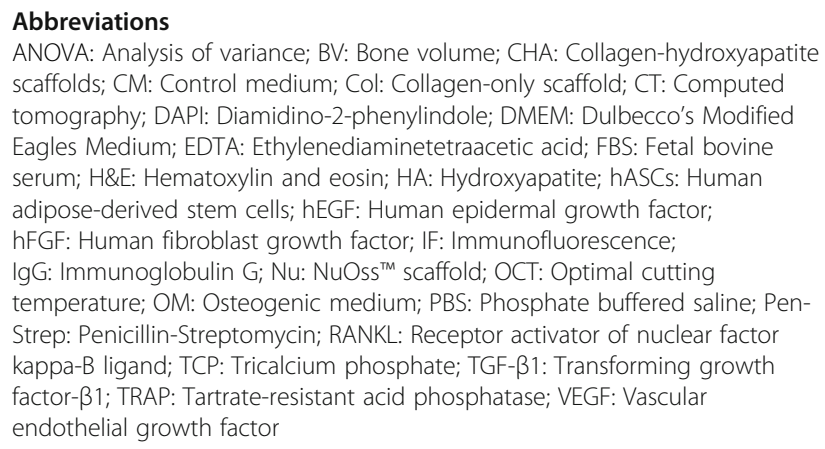
scaffolds; CM: Control medium; Col: Collagen-only scaffold; CT: Computed tomography; DAPI: Diamidino-2-phenylindole; DMEM: Dulbecco's Modified Eagles Medium; EDTA: Ethylenediaminetetraacetic acid; FBS: Fetal bovine serum; H\&E: Hematoxylin and eosin; HA: Hydroxyapatite; hASCs: Human adipose-derived stem cells; hEGF: Human epidermal growth factor; hFGF: Human fibroblast growth factor; IF: Immunofluorescence; IgG: Immunoglobulin G; Nu: NuOss ${ }^{\mathrm{TM}}$ scaffold; OCT: Optimal cutting temperature; OM: Osteogenic medium; PBS: Phosphate buffered saline; PenStrep: Penicillin-Streptomycin; RANKL: Receptor activator of nuclear factor kappa-B ligand; TCP: Tricalcium phosphate; TGF- $\beta 1$ : Transforming growth factor-B1; TRAP: Tartrate-resistant acid phosphatase; VEGF: Vascular endothelial growth factor

\section{Acknowledgements}

The authors acknowledge the Freimann Life Science Center at the University of Notre Dame for the care of animals.

\section{Authors' contributions}

HEW-B was involved in the study design, conducted all in vitro cell studies, lead the animal procedures, prepared histological and immunofluorescence images and performed the related analyses, and drafted the manuscript. MJM fabricated the collagen/hydroxyapatite scaffolds, participated in the animal procedures, and contributed to writing the manuscript. JAG conducted the micro-CT measurement and analysis, participated in the animal procedures, and contributed to writing the manuscript. GLN, RKR and DRW contributed to the study design, interpretation of the results, and revised the manuscript for publication. All authors read and approved the final manuscript.

\section{Funding}

This research was supported by the U.S. Army Medical Research and Materiel Command (W81XWH-09-1-0741) and was made possible in part by NIH R01 AR069657.

\section{Availability of data and materials}

The datasets used and/or analyzed during the current study are available from the corresponding author on reasonable request.

\section{Ethics approval and consent to participate}

Animal procedures were carried out in compliance with protocols approved by the Institutional Animal Care and Use Committee (IACUC) at the

University of Notre Dame.

\section{Consent for publication}

Not applicable.

\section{Competing interests}

The authors declare that they have no competing interests.

\section{Author details \\ ${ }^{1}$ Bioengineering Graduate Program, University of Notre Dame, Notre Dame, IN 46556, USA. ${ }^{2}$ School of Engineering, University of Mount Union, Alliance, $\mathrm{OH} 44601$, USA. ${ }^{3}$ Department of Aerospace and Mechanical Engineering, University of Notre Dame, Notre Dame, IN 46556, USA. ${ }^{4}$ Department of Mechanical and Energy Engineering, Indiana University-Purdue University Indianapolis, 723 W. Michigan Ave SL260, Indianapolis, IN 46202, USA.}

Received: 20 August 2019 Accepted: 30 September 2019 Published online: 17 October 2019

\section{References}

1. Komatsu DE, Warden SJ. The control of fracture healing and its therapeutic targeting: improving upon nature. J Cell Biochem. 2010;109:302-11. 
2. Giannoudis PV, Dinopoulos H, Tsiridis E. Bone substitutes: an update. Injury. 2005;36(Supplement 3):S20-27.

3. Burg KJLL, Porter S, Kellam JF. Biomaterial developments for bone tissue engineering. Biomaterials. 2000;21:2347-59.

4. Gleeson JP, Plunkett NA, O'Brien FJ. Addition of hydroxyapatite improves stiffness, interconnectivity and osteogenic potential of a highly porous collagen-based scaffold for bone tissue regeneration. Eur Cell Mater. 2010; 20:218-30.

5. Kane RJ, Roeder RK. Effects of hydroxyapatite reinforcement on the architecture and mechanical properties of freeze-dried collagen scaffolds. $J$ Mech Behav Biomed Mater. 2012;7:41-9.

6. Kane RJ, Weiss-Bilka HE, Meagher MJ, Liu Y, Gargac JA, Niebur GL, et al. Hydroxyapatite reinforced collagen scaffolds with improved architecture and mechanical properties. Acta Biomater. 2015;17:16-25.

7. Meagher MJ, Weiss-Bilka HE, Best ME, Boerckel JD, Wagner DR, Roeder RK. Acellular hydroxyapatite-collagen scaffolds support angiogenesis and osteogenic gene expression in an ectopic murine model: effects of hydroxyapatite volume fraction. J Biomed Mater Res - Part A. 2016;104: 2178-88.

8. Cao Y, Sun Z, Liao L, Meng Y, Han Q, Zhao RC. Human adipose tissuederived stem cells differentiate into endothelial cells in vitro and improve postnatal neovascularization in vivo. Biochem Biophys Res Commun. 2005; 332(2):370-9.

9. Scherberich A, Müller AM, Schäfer DJ, Banfi A, Martin I. Adipose tissuederived progenitors for engineering osteogenic and vasculogenic grafts. J Cell Physiol. 2010;225(2):348-53.

10. Hattori H, Masuoka K, Sato M, Ishihara M, Asazuma T, Takase B, et al. Bone formation using human adipose tissue-derived stromal cells and a biodegradable scaffold. J Biomed Mater Res B Appl Biomater. 2006;76(1): 230-9.

11. Hicok KC, Du Laney TV, Zhou YS, Halvorsen Y-DC, Hitt DC, Cooper LF, et al. Human adipose-derived adult stem cells produce osteoid in vivo. Tissue Eng. 2004;10(3-4):371-80.

12. Scherberich A, Galli R, Jaquiery C, Farhadi J, Martin I. Three-dimensional perfusion culture of human adipose tissue-derived endothelial and osteoblastic progenitors generates osteogenic constructs with intrinsic vascularization capacity. Stem Cells. 2007;25(7):1823-9.

13. Lv L, Liu Y, Zhang P, Zhang X, Liu J, Chen T, et al. The nanoscale geometry of TiO2 nanotubes influences the osteogenic differentiation of human adipose-derived stem cells by modulating H3K4 trimethylation. Biomaterials. 2015;39:193-205

14. Zhang Z, Luo X, Xu H, Wang L, Jin X, Chen R, et al. Bone marrow stromal cell-derived extracellular matrix promotes osteogenesis of adipose-derived stem cells. Cell Biol Int. 2015;39(3):291-9 [cited 2018 Aug 14]. Available from: http://doi.wiley.com/10.1002/cbin.10385.

15. Roberts SJ, Geris L, Kerckhofs G, Desmet E, Schrooten J, Luyten FP. The combined bone forming capacity of human periosteal derived cells and calcium phosphates. Biomaterials. 2011;32(19):4393-405.

16. Weiss HE, Roberts SJ, Schrooten J, Luyten FP. A semi-autonomous model of endochondral ossification for developmental tissue engineering. Tissue Eng Part A. 2012;18(13-14):1334-43.

17. Estes BT, Diekman BO, Gimble JM, Guilak F. Isolation of adipose-derived stem cells and their induction to a chondrogenic phenotype. Nat Protoc. 2010;5(7):1294-311.

18. Weiss-Bilka HE, McGann ME, Meagher MJ, Roeder RK, Wagner DR. Ectopic models for endochondral ossification: comparing pellet and alginate bead culture methods. J Tissue Eng Regen Med. 2018;12:e541-49.

19. Estes BT, Diekman BO, Guilak F. Monolayer cell expansion conditions affect the chondrogenic potential of adipose-derived stem cells. Biotechnol Bioeng. 2008;99:986-95

20. Hattori H, Sato M, Masuoka K, Ishihara M, Kikuchi T, Matsui T, et al. Osteogenic potential of human adipose tissue-derived stromal cells as an alternative stem cell source. Cells Tissues Organs. 2004;178(1):2-12.

21. Deuerling JM, Rudy DJ, Niebur GL, Roeder RK. Improved accuracy of cortical bone mineralization measured by polychromatic microcomputed tomography using a novel high mineral density composite calibration phantom. Med Phys. 2010;37(9):5138-45 [cited 2018 Sep 21]. Available from: http://doi.wiley.com/10.1118/1.3480507.

22. Mosiman V, Patterson B, Canterero L, Goolsby C. Reducing cellular autofluorescence in flow cytometry: an in situ method. Cytometry. 1997; 30(3):151-6
23. Domev H, Amit M, Laevsky I, Dar A, Itskovitz-Eldor J. Efficient engineering of vascularized ectopic bone from human embryonic stem cell-derived mesenchymal stem cells. Tissue Eng Part A. 2012;18(21-22):2290-302.

24. Selinummi J, Seppala J. Software for quantification of labeled bacteria from digital microscope images by automated image analysis. Biotechniques. 2005;39(6):859-63.

25. Wang Y, Wang Q, Haldar J, Yeh F, Xie M. Quantification of increased cellularity during inflammatory demyelination. Brain. 2011;134(Pt 12):3590-601.

26. Rathbone CR, Guda T, Singleton BM, Oh DS, Appleford MR, Ong JL, et al. Effect of cell-seeded hydroxyapatite scaffolds on rabbit radius bone regeneration. J Biomed Mater Res - Part A. 2014;102:1458-66.

27. Rai B, Lin JL, Lim ZXH, Guldberg RE, Hutmacher DW, Cool SM. Differences between in vitro viability and differentiation and in vivo bone-forming efficacy of human mesenchymal stem cells cultured on PCL-TCP scaffolds. Biomaterials. 2010:31:7960-70

28. Lyons FG, Al-Munajjed AA, Kieran SM, Toner ME, Murphy CM, Duffy GP, et al. The healing of bony defects by cell-free collagen-based scaffolds compared to stem cell-seeded tissue engineered constructs. Biomaterials. 2010;31: 9232-43.

29. Atkins GJ, Kostakis P, Pan B, Farrugia A, Gronthos S, Evdokiou A, et al. RANKL expression is related to the differentiation state of human osteoblasts. J Bone Miner Res. 2003:18:1088-98.

30. Denhardt D, Guo X. Osteopontin: a protein with diverse functions. FASEB J. 1993;7(15):1475-82.

31. Ferrara N, Davis-Smyth T. The biology of vascular endothelial growth factor. Endocr Rev. 1997;18(1):4-25.

32. Carano R, Filvaroff E. Angiogenesis and bone repair. Drug Discov Today. 2003:8(21):980-9.

33. Müller AM, Mehrkens A, Schäfer DJ, Jaquiery C, Güven S, Lehmicke M, et al. Towards an intraoperative engineering of osteogenic and vasculogenic grafts from the stromal vascular fraction of human adipose tissue. Eur Cell Mater. 2010;19(0):127-35.

34. Elabd C, Chiellini C, Massoudi A, Cochet O, Zaragosi L-E, Trojani C, et al. Human adipose tissue-derived multipotent stem cells differentiate in vitro and in vivo into osteocyte-like cells. Biochem Biophys Res Commun. 2007; 361(2):342-8.

\section{Publisher's Note}

Springer Nature remains neutral with regard to jurisdictional claims in published maps and institutional affiliations.

\section{Ready to submit your research? Choose BMC and benefit from:}

- fast, convenient online submission

- thorough peer review by experienced researchers in your field

- rapid publication on acceptance

- support for research data, including large and complex data types

- gold Open Access which fosters wider collaboration and increased citations

- maximum visibility for your research: over $100 \mathrm{M}$ website views per year

At $\mathrm{BMC}$, research is always in progress.

Learn more biomedcentral.com/submissions 